\title{
Measurement of spring constants of various spring-mass systems by using smartphones: A teaching proposal
}

\author{
Mustafa Erol a, *, Şeyda Kaya ${ }^{b}$, Kadir Hocaoğlu c \\ Dokuz Eylül University. Alsancak, No: 144 35210, Cumhuriyet Blv, 35220 Konak, Turkey \\ a mustafa.erol@deu.edu.tr, ${ }^{b}$ kaayaseyda@gmail.com, ${ }^{c}$ hocaoglu.k@gmail.com \\ * Corresponding Author.
}

Received: 17 February 2020; Revised: 21 March 2020; Accepted: 31 March 2020

\begin{abstract}
This study aims to develop a teaching proposal to measure spring constants of various spring-mass systems by means of the smartphones. Specifically, a single springmass system, a serial connected and a parallel connected spring systems are experimentally resolved, by using the ambient light sensor of the smartphones. The measurements are achieved by simply recording the light intensity, detected by the oscillating smartphone, as a function of time for the simple harmonic motion. Using the light intensity-time graphs, the average periods and eventually the spring constants are estimated and the outcomes are compared with the theoretical results. The overall outcomes of the work indicate some 3,3\% relative error for the serial connected springs and $10,8 \%$ relative error for the parallel connected springs. The approach is important in the sense that the apparatus directly plots instantaneous momentum-time graphs and it creates an enjoyable and beneficial teaching atmosphere.
\end{abstract}

Keywords: physics education; parallel spring system; serial spring system; simple harmonic motion; smart phones.

How to Cite: Erol, M., Kaya, Ş., \& Hocaoğlu, K. (2020). Measurement of spring constants of various spring-mass systems by using smartphones: A teaching proposal. Momentum: Physics Education Journal, 4(1), 1-10. https://doi.org/10.21067/mpej.v4i1.4150

\section{Introduction}

Physics tries to resolve the nature and natural phenomena that contain very complicated concepts and equations. This is why, there are certain difficulties in teaching physical concepts and laws, hence developing an improved understanding and maintaining continuous progress are very challenging tasks for the physics educators.

In today's rapidly advancing technologies, besides the acquisition of knowledge, understanding the nature of science, producing scientific knowledge, understanding what is happening around us and producing solutions to problems are amongst the main objectives of Physics Education Research (PER). There are many teaching models based on experiments concerning science education and experimentation should always be at the heart of the science teaching. For instance, Petry itemized that, scientific knowledge, taught without any laboratory work, makes no sense to the majority of the students (Petry et al., 2016).

Concerning technology assisted education, the interaction of the learner with the aimed knowledge, is maintained by the appropriate technology hence, the technology provides the opportunity to intervene and direct the learning processes. The number of sensors, in today's smartphones, rapidly increases day by day and accordingly the smartphones can be seen as miniature tool boxes with very rich sensors, beneficial and suitable for difficult teaching tasks. It is understandable that when the phone manufacturers have placed the sensors, some of which are light sensors, proximity sensors, accelerometers, gyroscopes, barometers, and magnetometers, they have most likely not 
anticipated the educational and scientific purposes. Nevertheless, these portable tool boxes lead to a new route to measure many concepts in physics education (Díaz-Melián et al., 2018), to give some examples, in astronomy (Barrera-Garrido, 2015), thermodynamics (Monteiro \& Martí, 2016), magnetism (Taspika et al., 2018) and optics (Sans et al., 2016).

Although the resolution of simple pendulum seems easy, it is a subject with convinced difficulties in itself. In order to mention a few, vibration characteristics of a cantilever plate with attached spring-mass system is resolved by Chiba and Sugimoto (2003). The resolution of some complicated spring-mass systems is handled by Cushing (1984). The visualization of the oscillation and understanding of the concept of spring constant can sometimes be incomprehensible to majority of the students. Measuring spring constants with a vertical spring mass oscillator is a standard high school laboratory activity. Traditionally, the oscillation period of the spring system is measured with a fault-prone stopwatch due to the adverse effect of the reaction time of the experimenter (Pili, 2018). The use of smartphones, in elementary experimental physics, has been a popular activity and existed for a number of years (Çoban \& Erol, 2019; Countryman, 2014; Dilek \& Erol, 2018; Erol \& Çolak, 2018). The smartphone light sensors also find room for the application opportunities in experimental kinematics, such as the measurement of velocity and acceleration and free damping oscillations (Pramudya \& Raharja, 2019; Sans et al., 2013).

In this study, we present a teaching tool to measure the spring constants of various spring mass systems by means of the smartphones. The resolution of the simple harmonic motion is managed by directly obtaining the light intensity-time graphs from the physics tool box application. The measured experimental data are compared with the theoretical outcomes and a fairly good agreement is detected.

The spring pendulum is a very important and simple instrument to demonstrate and teach the simple harmonic motion. When a mass of $m$ is attached to the bottom of a vertically mounted spring and the mass is displayed from the equilibrium position by an amount of $x$, causes the simple harmonic motion to commence. The spring-mass system moves ceaselessly, assuming the air and contact frictions are negligible. Resolution of the motion or oscillations, assuming that the actual spring stays within the limits of the flexibility, is straightforward and the oscillations simply obey to the Newton's second law, that is, $m \frac{d^{3} x}{d t^{2}}=-k x$, (Serway \& Jewett, 2018). Knowing that the harmonic motion is described by the equation of, $\frac{d^{2} x}{d t^{2}}=-w^{2} x$, then the period of the oscillation can be expressed by,

$T=2 \pi \sqrt{\frac{m}{k}}$

Assuming that two separate springs with spring constants of $k_{1}$ and $k_{2}$ are connected in serial, then the equivalent spring constant of the resulting compound spring system is given by,

$k_{e q}=\frac{k_{1}, k_{2}}{k_{1}+k_{2}}$

Similarly, when two springs with spring constants of $k_{1}$ and $k_{2}$ are connected in parallel, then the equivalent spring constant of the resulting compound spring system is given by,

$k_{\text {eq }}=k_{1}+k_{2}$

These expressions are obviously valid in an ideal case, in other words the springs oscillate within the limits of the flexibility and the contact and air friction effects are negligibly small. One can smoothly resolve and test these theoretical equations by experimentally determining the actual oscillation periods and estimating the spring constants of various spring-mass systems. 
Momentum: Physics Education Journal, 4 (1), 2020, 3

Mustafa Erol, Şeyda Kaya, Kadir Hocaoğlu

\section{Methods}

The apparatus is comprised of a spring pendulum with a smartphone is mounted at the bottom and a second smartphone with the light sensor application is on, specifically set to plot the actual intensity time graph. The apparatus is shown in the Figure 1. The smartphones employed in the present work are ordinary in the sense that they are embedded with a light and a motion sensor together with the required physics toolbox sensor suit software.

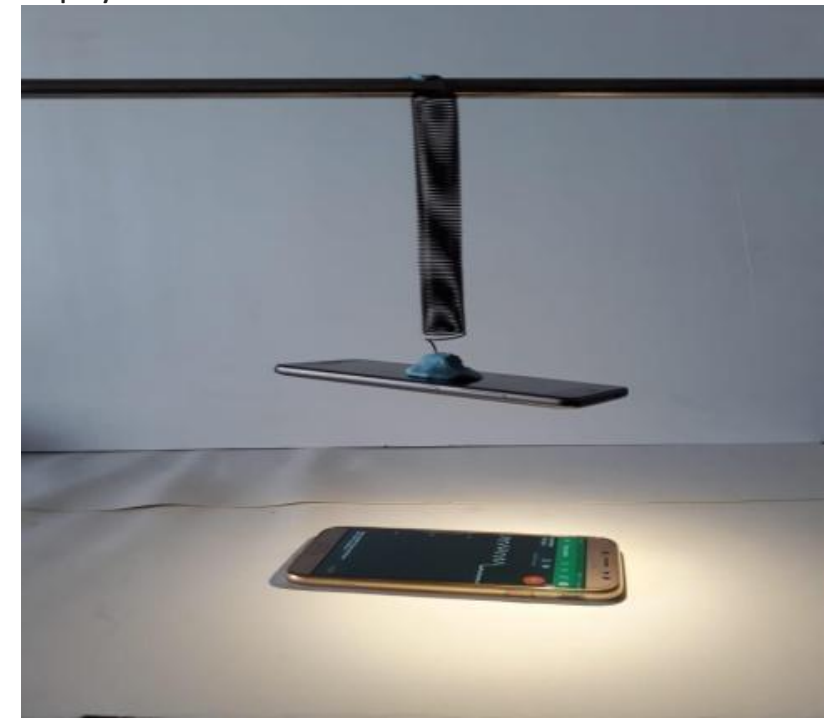

Figure 1. The photography of the basic apparatus, comprised of a spring pendulum with an oscillating smartphone as the actual mass and a stationary second smartphone.

The spring mass system is built such that the oscillating mass of the pendulum is a smartphone with the light sensor facing down and adjusted on the same vertical line such that the light intensity can be measured at uppermost sensitivity. The experimentations are managed by switching on the physics toolbox suite and commencing the simple harmonic motion for the mass/phone mounted to the actual spring. Simultaneously the posometer of the smartphone on the table is turned on and resulting light intensity - time graphs are recorded and displayed. Naturally the light intensity-time graphs are separately obtained for the two separate single springs, and also for the serial connected and parallel connected spring systems.

In order to perform properly and to get best results, some important points should be taken into account when conducting the experiments. The first point is the accurate adjustment of the luminous oscillating smartphone to match the sensor of the stationary smartphone. Basically, if the sensor of the stable phone does not coincide well enough with the oscillating phone then the light intensity - time graph does not come out accurately. Secondly, if the oscillating smartphone is not arranged to have very small oscillations, then the system immediately becomes unstable and cannot make smooth harmonic motion. Therefore, it is best to oscillate the system with oscillation amplitudes not exceeding $1 \mathrm{~cm}$. Finally, as the system inevitably begins to fade after a while, the measurements must be performed before the fading starts.

\section{Physics toolbox sensor suite}

Physics Toolbox Sensor Suite is an application, comprising a large number of measurement sensors and many crucial physical concepts which can directly and precisely be monitored and determined. The screenshot of the actual application is shown in the Figure 2. 
Momentum: Physics Education Journal, 4 (1), 2020, 4

Mustafa Erol, Şeyda Kaya, Kadir Hocaoğlu

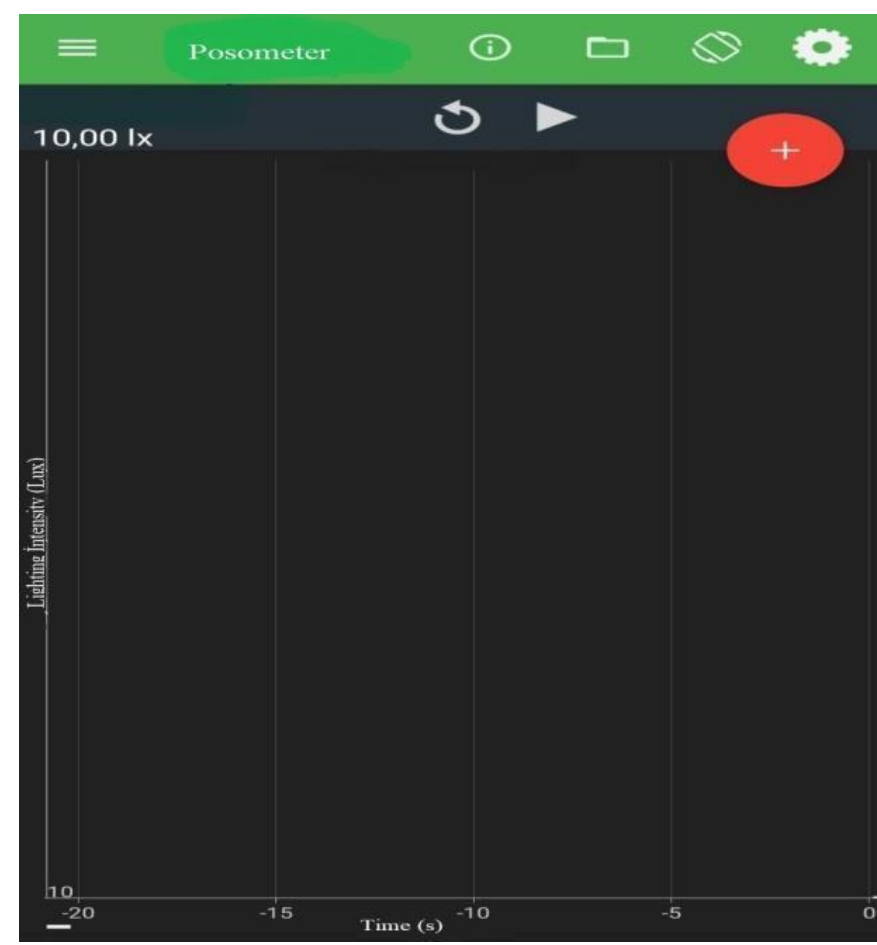

Figure 2. The screenshot of the physics toolbox sensor suite application.

In the application, one can measure central concepts such as pressure, sound intensity, light intensity, magnetic field and position and one can also monitor the relating graphs of the data selected within the application. The application can carry out complicated experiments and retrieve the data in a more practical way without having any additional measurement tools (Monteiro \& Martí, 2016; Pili, 2018; Pili \& Violanda, 2019). In the present work, the posometer sensor of the physics toolbox sensor suite is employed and basically the illumination intensity coming from the light source is plotted as a function of time with the help of the posometer sensor.

\section{Results and Discussions}

\section{Single spring case}

In order to measure the spring constant of the selected first spring, the simple spring pendulum, shown in the Figure 1 is basically equipped and employed. The first smartphone, employed as the actual mass of the pendulum and attached to the bottom of the spring, is moved from the equilibrium position by an amount of about $1 \mathrm{~cm}$ and the system let to oscillate freely. The first smartphone, with an oscillating mass, is also used as the genuine light source and the second smartphone fixed appropriately on the table and is set to measure the light intensity oscillations by using embedded light detector. Using the special application of the physics tool box, the light intensity-time graph of the oscillation is recorded and displayed directly by the fixed smartphone. Typical recorded light intensity-time graph of the oscillation is shown the Figure 3 , as the screenshot. 


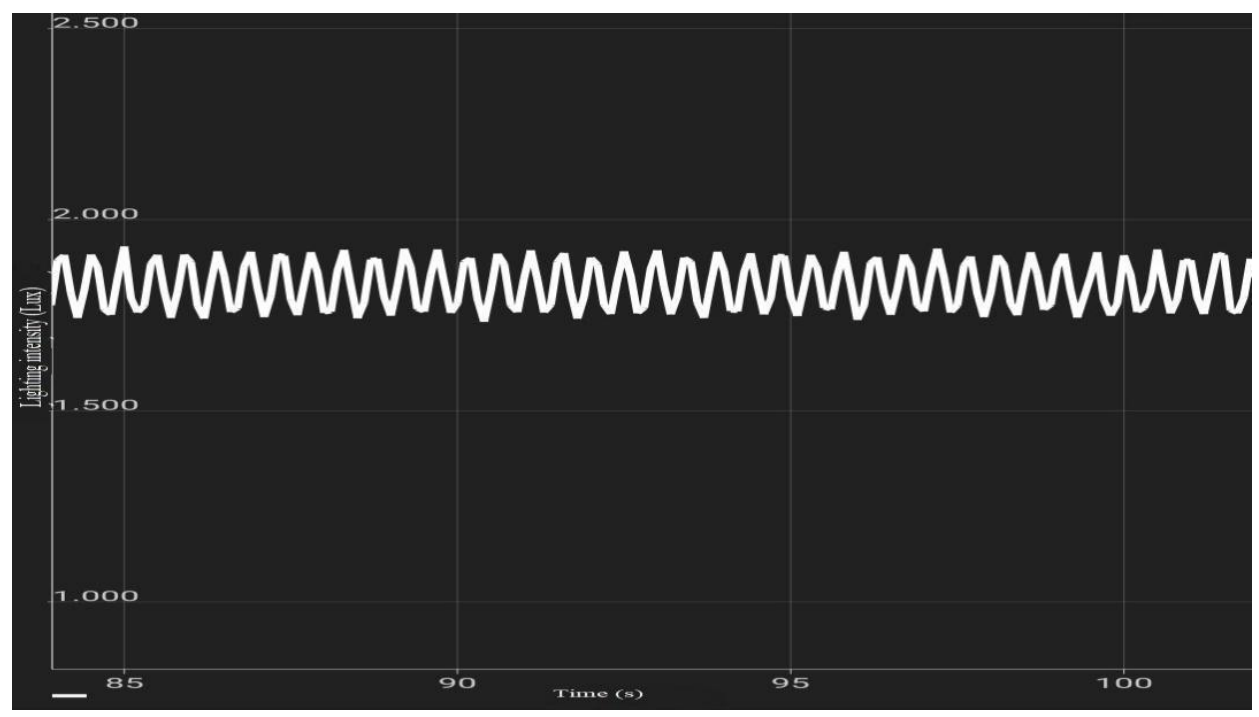

Figure 3. The screenshot of the first single spring, clearly showing the simple harmonic motion of the spring mass system.

The average period, $T_{0}$, of the motion is directly measured from this graph, by basically reading the number of oscillations per 5 seconds and by dividing the overall evolved time to the number of periods. The number of oscillations within the time interval of $5 \mathrm{~s}$ is measured 3 times and the experimental data and the calculated period values are given in the Table 1.

Table 1. The outcomes of the first spring mass system, showing the measurement time intervals, number of periods within the time intervals and the average oscillation period.

\begin{tabular}{ccc}
\hline$\Delta t(s)$ & $\mathrm{T}_{\mathrm{n}}$ (number of periods) & $\mathrm{T}(\mathrm{s})$ \\
\hline $85-90$ & 10,7 & 0,467 \\
$90-95$ & 10,3 & 0,485 \\
$95-100$ & 10,8 & 0,462 \\
& & $\mathrm{~T}_{0}=0,471 \mathrm{~s}$ \\
\hline
\end{tabular}

The mean value of the oscillation period is calculated to be $0,471 \mathrm{~s}$. Using the overall oscillating mass, that is solely the smartphone with a mass of $m=191,3 \mathrm{~g}$, it is direct to calculate the spring constant from the equation of,$k_{1}{ }^{2}=\frac{4 \pi^{3} m}{T_{0}{ }^{3}}$, and the final result for the spring constant is found to be, $k_{1}=33,98 \mathrm{~N} / \mathrm{m}$.

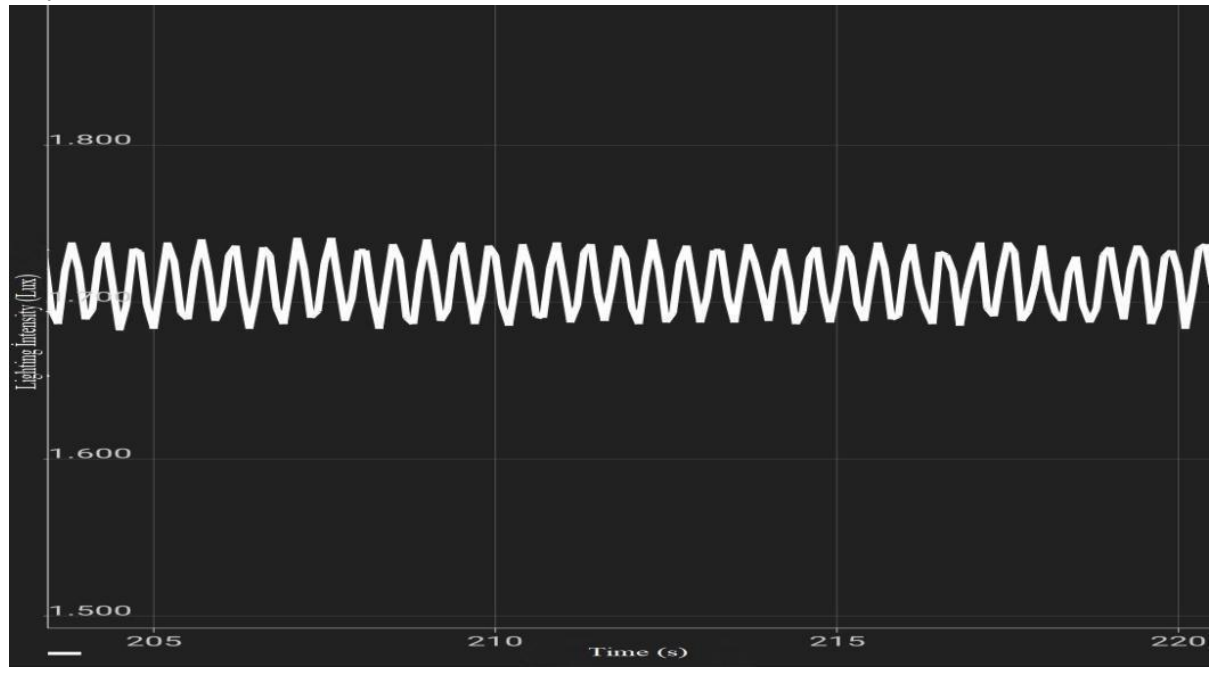

Figure 4. The oscillation screenshot of the second spring, noticeably showing the simple harmonic motion of the spring -mass system. 
In order to estimate the spring constant of the second spring, exactly same procedure is carried out by only changing the actual spring. In this case, the light intensity, determined as a function of time for the free oscillations and the typical screenshot of the oscillations is given in the Figure 4.

The same method is employed to estimate the average oscillation period of the motion and actual outcomes of the measurements are given in the Table 2 . In this case, the average period is estimated to be, $T_{0}=0,473 \mathrm{~s}$. Using the overall oscillating mass, that is $m=191,3 \mathrm{~g}$, and the equation of, $k_{2}{ }^{2}=\frac{4 \pi^{3} m}{T_{0}{ }^{2}}$, eventually leads to the spring constant of, $k_{2}=33,67 \mathrm{~N} / \mathrm{m}$.

Table 2. The outcomes of the second spring mass system, showing the measurement time intervals, measured number of periods within the time intervals and the calculated average oscillation period.

\begin{tabular}{ccc}
\hline$\Delta t(s)$ & $\mathrm{T}_{\mathrm{n}}$ (number of periods) & $\mathrm{T}(\mathrm{s})$ \\
\hline $205-210$ & 10,7 & 0,467 \\
$210-215$ & 10,5 & 0,476 \\
$215-220$ & 10,5 & 0,476 \\
& & $\mathrm{~T}_{0}=0,473 \mathrm{~s}$ \\
\hline
\end{tabular}

The results obtained from two distinct approaches are in a very good agreement with each other and overall method is well-matched with the approach offered by Pili (2018).

\section{Serial Connection}

One of the central objectives of this work is to resolve the oscillations of the serial and parallel connected spring systems. The basic apparatus used to measure the spring constant of the serially connected spring system is shown in the Figure 5 . The two springs, with previously measured spring constants, are employed in this case and the actual connection point between the springs is particularly made very stiff to prevent any loosening.

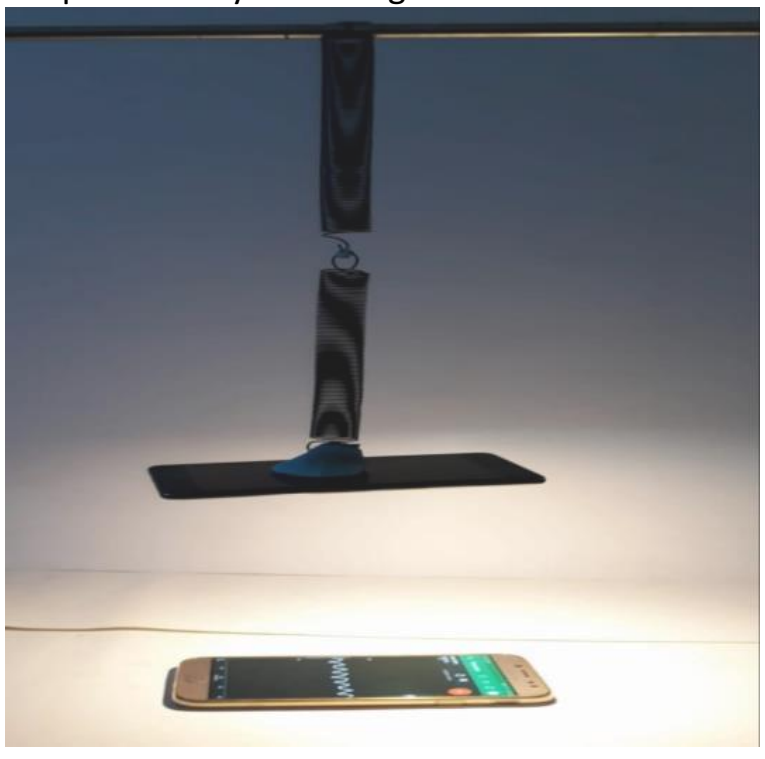

Figure 5. The photography of the serial connected spring-mass system.

Typical oscillation graph, in other words the light intensity versus time graph, is given in the Figure 6. The screenshot picture clearly shows smooth and discrete oscillations which can be used to experimentally measure the average oscillation period. 


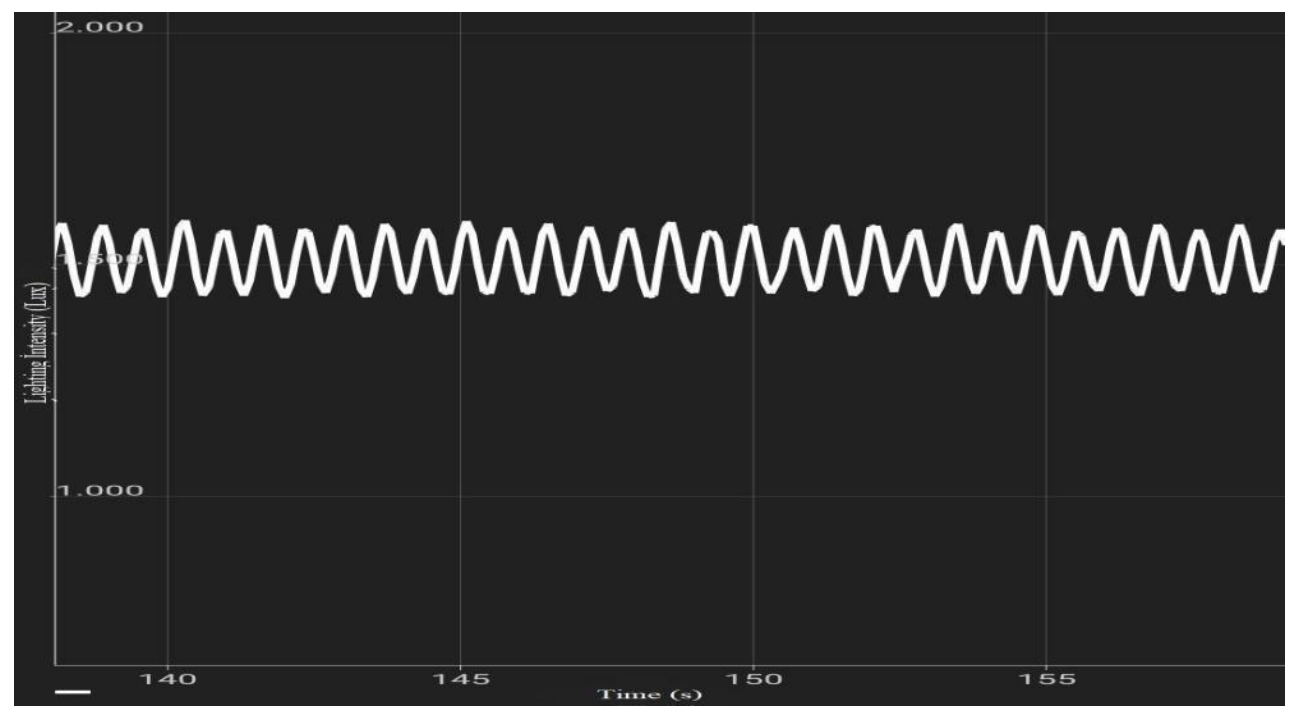

Figure 6. The oscillation screenshot of the serial connected spring system, noticeably showing the simple harmonic motion of the spring -mass system.

In order to measure the average period, the oscillations between the time interval of 140s155 s, are taken into account. The experimental results are given in the Table 3.

Table 3. The outcomes of the serially connected spring mass system, showing the measurement time intervals, measured number of periods within the time interval and the calculated average oscillation period.

\begin{tabular}{ccc}
\hline$\Delta \boldsymbol{t}(\boldsymbol{s})$ & $\mathrm{T}_{\mathrm{n}}$ (number of periods) & $\mathrm{T}(\mathrm{s})$ \\
\hline $140-145$ & 7,8 & 0,641 \\
$145-150$ & 7 & 0,714 \\
$150-155$ & 7,3 & 0,684 \\
& & $\mathrm{~T}_{0}=0,679 \mathrm{~s}$ \\
\hline
\end{tabular}

In order to extract the equivalent spring constant for the serially connected spring mass system, the data in the Table 3 is basically used. It is straightforward to measure the average oscillation period which is estimated to be, $T_{0}=0,679 \mathrm{~s}$. Using the oscillation mass of, $m=191,3 \mathrm{~g}$, and the formula for the equivalent spring constant, $k_{e q}{ }^{2}=\frac{4 \pi^{2} m}{T_{0}^{2}}$, gives the final result for the serial spring system as, $\mathrm{k}_{\mathrm{eq}}=16,36 \mathrm{~N} / \mathrm{m}$. In order to compare this experimental result with the theoretical estimation, the equivalent spring constant is also calculated from the theoretical equation. Using the previously calculated separate spring constants of $k_{1}=33,98 \mathrm{~N} / \mathrm{m}$ and $k_{2}=33,67 \mathrm{~N} / \mathrm{m}$, one can easily estimate the equivalent spring constant and that leads to the final result of $k_{e q}=\frac{k_{1} \cdot k_{2}}{k_{1}+k_{2}}=16,91 \mathrm{~N} / \mathrm{m}$. The discrepancy between the experimental and theoretical analysis is about, \%3,3, and this relative error seems to be quite reasonable within the limits of the experimental sensitivity. The inconsistency between the theoretical result and the experimental result is mainly attributed to the design of the spring-mass system and the environmental effects, such as air and contact frictions. The results are quite compatible with the previous approach of (Pili \& Violanda, 2019).

\section{Parallel connection}

The apparatus, employed to measure the spring constant for the parallel connected spring system, is shown in the Figure 7. In this case, a special effort is spent to adjust the oscillating smartphone perfectly horizontal and also such that the light intensity variation due to the motion can be detected and recorded healthy. 
Momentum: Physics Education Journal, 4 (1), 2020, 8

Mustafa Erol, Şeyda Kaya, Kadir Hocaoğlu

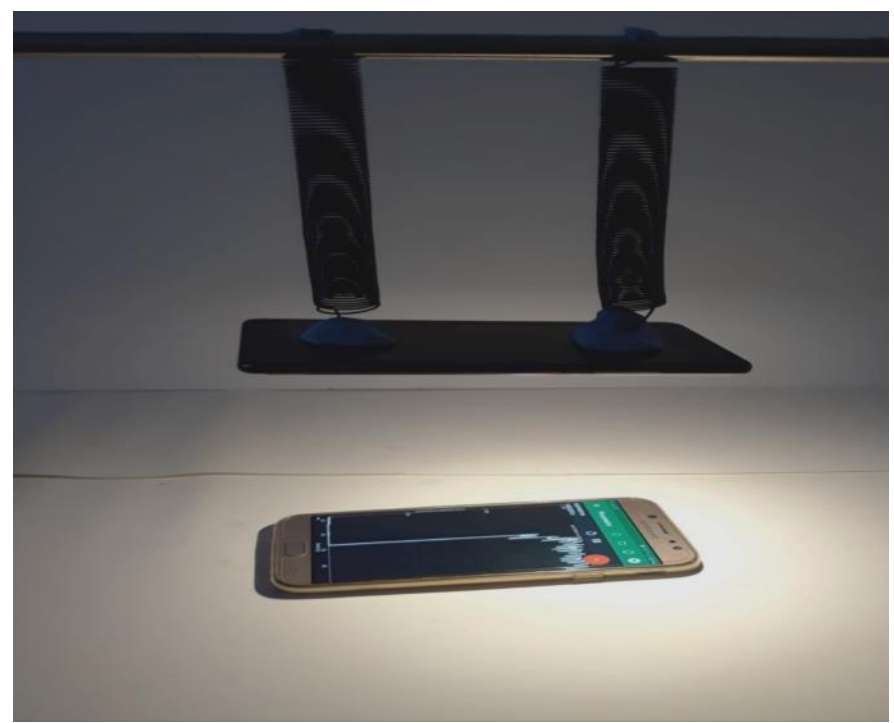

Figure 7. The photography of the parallel connected spring-mass system with two smartphones.

The light intensity plotted as a function of time, during the smooth oscillations, is recorded by the employed physics tool box and the typical oscillation graph is given in the Figure 8 .

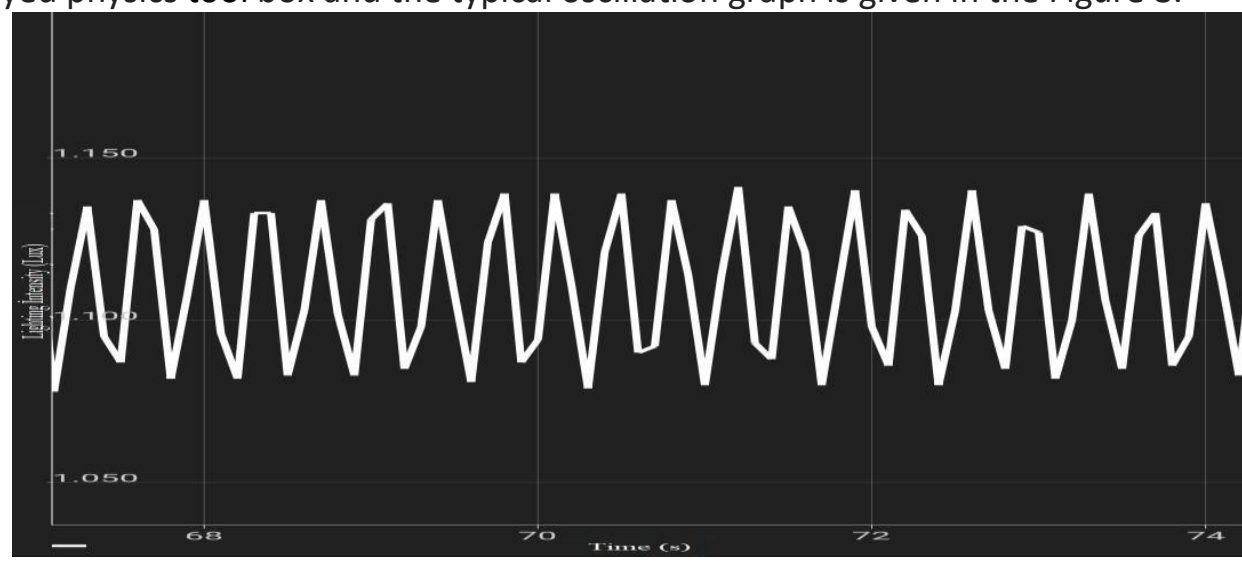

Figure 8. The oscillation screenshot of the parallel connected spring system, noticeably showing the simple harmonic motion of the spring -mass system.

In order to measure the average period, the oscillations between 140s and 155s are basically considered. The outcomes are given in the Table 4.

Table 4. The outcomes of the parallel connected spring mass system, showing the measurement time intervals, measured number of periods within the time interval and calculated average oscillation periods.

\begin{tabular}{ccc}
\hline$\Delta \boldsymbol{t}(\boldsymbol{s})$ & $\mathrm{T}_{\mathrm{n}}$ (number of periods) & $\mathrm{T}(\mathrm{s})$ \\
\hline $68-70$ & 5,7 & 0,351 \\
$70-72$ & 5,6 & 0,357 \\
$72-74$ & 5,7 & 0,351 \\
& & $\mathrm{~T}_{0}=0,353 \mathrm{~s}$ \\
\hline
\end{tabular}

In order to estimate the equivalent spring constant for the parallel connected spring mass system, the data in the Table 4 is employed. The average oscillation period, in this case, is estimated to be, $T_{0}=0,353 \mathrm{~s}$. Substitution of the oscillation mass of, $m=191,3 \mathrm{~g}$, and the average period in the expression for the equivalent spring constant, $k_{e q}{ }^{2}=\frac{4 \pi^{2} m}{T_{0}{ }^{2}}$, leads to the final result of, $k_{\text {eq }}=60,35$ $\mathrm{N} / \mathrm{m}$. In order to compare this experimental result with the theoretical estimation, the equivalent spring constant is also calculated from the theoretical equation. Using the previously calculated separate spring constants of, $k_{1}=33,98 \mathrm{~N} / \mathrm{m}$ and $k_{2}=33,67 \mathrm{~N} / \mathrm{m}$, leads to the final result of, 
$k_{e q}=\frac{k_{1}, k_{z}}{k_{1}+k_{z}}=67,65 \mathrm{~N} / \mathrm{m}$. The discrepancy between the experimental and theoretical analysis is larger in this case and is about, \%10,8. The difference is assumed to be reasonable again within the limits of the experimental sensitivity. The inconsistency between the theoretical and experimental results could mainly be due to the design and the environmental influences, such as friction and connection problems. The overall evaluation of results leads to a very good agreement with the previous results of (Pili \& Violanda, 2019).

\section{Conclusions}

In this work, a simple and easily accessible teaching apparatus and method are developed and suggested, in order to resolve and understand the simple harmonic motion and also the motion of the various spring mass systems. The approach basically employs various spring mass systems with two smartphones. The measurements are achieved by quantifying and recording the light intensitytime graphs, in the sense that from the oscillations the periods and eventually the spring constants are estimated for serially and parallel connected spring systems. The measurements are compared with the theoretical analysis and reasonably good agreements are obtained. The approach is beneficial for physics teachers because of being an uncomplicated, easily reachable method and the approach offers an enjoyable physics education atmosphere.

\section{References}

Barrera-Garrido, A. (2015). Analyzing planetary transits with a smartphone. The Physics Teacher, 53(3), 179-181. https://doi.org/10.1119/1.4908091

Chiba, M., \& Sugimoto, T. (2003). Vibration characteristics of a cantilever plate with attached springmass system. Journal of Sound and Vibration, 260(2), 237-263. https://doi.org/10.1016/S0022460X(02)00921-5

Çoban, A., \& Erol, M. (2019). Teaching and determination of kinetic friction coefficient using smartphones. Physics Education, 54(2), 025019. https://doi.org/10.1088/1361-6552/aaff84

Countryman, C. L. (2014). Familiarizing students with the basics of a smartphone's internal sensors. The Physics Teacher, 52(9), 557-559. https://doi.org/10.1119/1.4902204

Cushing, J. T. (1984). The spring-mass system revisited. American Journal of Physics, 52(10), 925-933. https://doi.org/10.1119/1.13796

Díaz-Melián, V. L., Rodríguez, L. A., Pedroso-Camejo, F., Mieres, J., de Armas, Y., Batista-Leyva, A. J., \& Altshuler, E. (2018). Optics undergraduate experiments using smart (and not so smart) phones. In arXiv preprint arXiv:1811.09546.

Dilek, U., \& Erol, M. (2018). Detecting position using ARKit. Physics Education, 53(2), 25011. https://doi.org/10.1088/1361-6552/aaa0e6

Erol, M., \& Çolak, i. Ö. (2018). Magnetically driven oscillator and resonance: a teaching tool. Physics Education, 53(3), 35027. https://doi.org/10.1088/1361-6552/aab30b

Monteiro, M., \& Martí, A. C. (2016). Using smartphone pressure sensors to measure vertical velocities of elevators, stairways, and drones. In arXiv preprint arXiv:1607.00363.

Petry, C. A., Pacheco, F. S., Lohmann, D., Correa, G. A., \& Moura, P. (2016). Project teaching beyond Physics: Integrating Arduino to the laboratory. 2016 Technologies Applied to Electronics Teaching (TAEE), 1-6. https://doi.org/10.1109/TAEE.2016.7528376

Pili, U. (2018). A dynamic-based measurement of a spring constant with a smartphone light sensor. Physics Education, 53(3), 33002. https://doi.org/10.1088/1361-6552/aaa927

Pili, U., \& Violanda, R. (2019). Measuring a spring constant with a magnetic spring-mass oscillator and a telephone pickup. Physics Education, 54(4), 43001. https://doi.org/10.1088/1361$6552 / a b 1432$ 
Pramudya, Y., \& Raharja, E. (2019). The influence of inclined plane angle to the oscillation period of spring and block systems. Proceedings of the 2019 Ahmad Dahlan International Conference Series on Engineering and Science (ADICS-ES 2019). https://doi.org/10.2991/adics-es-19.2019.3

Sans, J. A., Gea-Pinal, J., Gimenez, M. H., Esteve, A. R., Solbes, J., \& Monsoriu, J. A. (2016). Determining the efficiency of optical sources using a smartphone's ambient light sensor. European Journal of Physics, 38(2), 25301. https://doi.org/10.1088/1361-6404/aa51a9

Sans, J. A., Manjón, F. J., Pereira, A. L. J., Gomez-Tejedor, J. A., \& Monsoriu, J. A. (2013). Oscillations studied with the smartphone ambient light sensor. European Journal of Physics, 34(6), 13491354. https://doi.org/10.1088/0143-0807/34/6/1349

Serway, R. A., \& Jewett, J. W. (2018). Physics for scientists and engineers with modern physics. Cengage learning.

Taspika, M., Nuraeni, L., Suhendra, D., \& Iskandar, F. (2018). Using a smartphone's magnetic sensor in a low-cost experiment to study the magnetic field due to Helmholtz and anti-Helmholtz coil. Physics Education, 54(1), 15023. https://doi.org/10.1088/1361-6552/aaefb4 